\title{
Raccourcissement de la durée de l'émission harmonique par génération d'une porte temporelle
}

\author{
M. Kovacev, H. Merdji, E. Priori ${ }^{1}$, Y. Mairesse, P. Monchicourt, P. Breger, \\ O. Tcherbakoff ${ }^{2}$, E. Mével ${ }^{2}$, E. Constant ${ }^{2}$, P. Salières, B. Carré et P. Agostini \\ Service des Photons, Atomes et Molécules, CEA-Saclay, 91191 Gif-sur-Yvette, France \\ 1 INFM, Dipartimento di Fisica, Politecnico, piazza Leonardo da Vinci 32, 20133 Milano, Italie \\ ${ }^{2}$ CELIA, Université Bordeaux I, 351 cours de la Libération, 33405 Talence cedex, France
}

\begin{abstract}
Résumé. Nous présentons la démonstration de principe d'une technique optique proposée pour diminuer la durée de l'émission harmonique générée par des impulsions laser "longues", constituées de plusieurs cycles optiques. En faisant varier l'ellipticité pendant l'impulsion laser, on peut confiner l'émission harmonique à la durée pendant laquelle la polarisation est quasi-linéaire. Les résultats obtenus et leur comparaison avec un modèle simple permettent de conclure que nous avons obtenu un raccourcissement d'un facteur 3 pour les harmoniques du plateau. L'utilisation d'impulsions laser plus courtes, de15-20 fs, devrait permettre d'isoler une seule impulsion de durée sub-femtoseconde.
\end{abstract}

\section{INTRODUCTION}

Il a récemment été démontré que, dans des conditions appropriées, les harmoniques d'ordre élevé d'une impulsion laser intense sont émises sous la forme d'un train d'impulsions attosecondes $(\sim 250$ as) séparées par une demi-période du fondamental (1,35 fs dans le cas d'un laser Titane:saphir) [1]. Cette structure temporelle s'explique facilement dans le cadre d'un modèle semiclassique [2,3], qui considère la génération des harmoniques (HHG) comme un processus en trois étapes: l'ionisation par effet tunnel d'un atome dans le champ laser intense, l'accélération de l'électron par le même champ et sa recombinaison radiative avec l'ion parent. Des impulsions attosecondes sont émises à chaque recollisions électron-ion, donc à chaque demi-période laser.

Un simple calcul classique permet de se rendre compte que, si la polarisation du laser n'est pas linéaire, la probabilité de recollision de l'électron avec son ion parent, et donc l'intensité de l'émission harmonique, décroît rapidement quand l'ellipticité $\varepsilon$ de l'impulsion laser augmente. Les mesures expérimentales $[4,5]$ ont montré que l'efficacité de génération décroît suivant la loi empirique $\mathrm{I}_{\mathrm{q}} \propto \mathrm{e}^{-\beta_{4} \varepsilon^{2}}$ où $I_{q}$ est l'intensité de l'harmonique d'ordre $\mathrm{q}$ et $\beta_{q}$ une constante qui augmente avec $q$ et dont la valeur est typiquement comprise entre 15 et 60 . Cela a conduit $P$. B. Corkum à suggérer d'utiliser une impulsion laser dont le degré d'ellipticité varie rapidement dans le temps pour définir une «porte temporelle » qui permettrait d'interdire toutes les recollisions sauf une: il en résulterait une impulsion attoseconde isolée [6]. Une impulsion isolée a récemment été obtenue par l'équipe de F. Krausz [7] sans avoir recours à une porte temporelle, mais simplement en utilisant un impulsion laser d'une durée de 7 fs, obtenue par la technique de la fibre creuse [8]. Cependant cette méthode présente certains inconvénients: elle est relativement difficile à maîtriser et l'énergie des impulsions lasers est limitée à moins d' $1 \mathrm{~mJ}$; de plus elle requiert de sélectionner seulement les harmoniques les plus élevées, pour lesquelles l'efficacité de génération est faible.

L'étude du confinement temporel par modulation de la polarisation garde donc tout son intérêt. Une première tentative en ce sens a été effectuée par le groupe de Lund [9], qui a réalisé une 
porte temporelle de $5 \mathrm{fs}$ en utilisant deux impulsions laser modulées en fréquence («chirpées»). Cependant cette méthode ne permet pas un contrôle fin de la durée de la porte et ne semble pas adaptée à la génération d'impulsions attosecondes. Nous présentons ici la réalisation expérimentale d'une technique, proposée indépendamment par E. Constant [10] et par V. T. Platonenko et V. V. Strelkov [11] qui, au contraire, permet de faire varier de manière continue la durée de l'impulsion harmonique, en principe jusqu'à des valeurs inférieures à $1 \mathrm{fs}$. Une première démonstration de cette technique mais avec un délai fixe a été récemment effectuée au laboratoire CELIA [12].

\section{PRINCIPE DE LA METHODE}

Cette méthode consiste à générer deux impulsions laser de même intensité, polarisées circulairement droite et gauche et décalées temporellement d'un délai $\tau$. Comme montré en Fig. 1, on obtient ainsi une impulsion dont la polarisation passe de circulaire à elliptique, puis à linéaire et qui ensuite redevient elliptique puis circulaire. La porte temporelle, qui peut être définie comme la durée pendant laquelle l'ellipticité est inférieure à un seuil fixé (par exemple 0,1), est d'autant plus étroite que le délai $\tau$ est important.

Le dispositif expérimental que nous avons utilisé comporte un interféromètre de Michelson, qui permet d'obtenir deux impulsions laser décalées d'un délai $\tau$ variable. Dans chacun de ses bras est insérée une lame biréfringente $\lambda / 4$; si les axes optiques des lames sont orientés à $\pm 45^{\circ}$ par rapport à la direction de polarisation du laser à l'entrée de l'interféromètre, les deux impulsions obtenues à la sortie sont polarisées linéairement suivant des directions orthogonales, car chaque lame quart d'onde est traversée deux fois, et agit donc comme une lame $\lambda / 2$. Ces deux impulsions traversent ensuite une troisième lame $\lambda / 4$ dont l'axe optique est orienté suivant la bissectrice des directions de polarisation. Elle transforme les deux polarisations linéaires en circulaires droite et gauche respectivement. En faisant varier le délai entre les deux impulsions, on modifie la durée de l'émission harmonique. Le faisceau laser qui sort du Michelson est focalisé à $7,5 \cdot 10^{13} \mathrm{~W} / \mathrm{cm}^{2}$, f\#52, dans un jet d'Argon de pression 50 Torr et le rayonnement harmonique est analysé par un spectromètre UVX constitué d'un miroir torique et d'un réseau plan en incidence rasante.
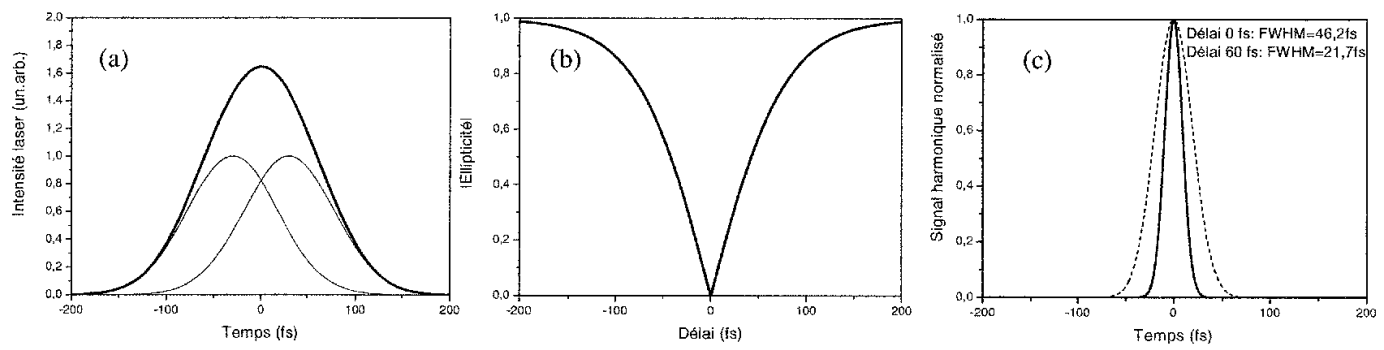

Figure 1, (a) profil temporel des deux impulsions laser polarisées circulairement et de l'impulsion résultant de leur superposition. (b) variation de l'ellipticité en fonction du temps. (c) profil temporel, calculé comme décrit dans la section 3 , de la $15^{\text {ème }}$ harmonique pour $\tau=0$ (pointillés) et $\tau=60$ fs (trait plein).

\section{RESULTATS}

La courbe en trait plein de la Fig.2 représente la dépendance en délai du flux de l'harmonique 15 générée par une impulsion laser de $80 \mathrm{fs}$. La modulation rapide de la trace est due au fait que, lorsque $\tau$ varie d'une demi-période optique, le plan de polarisation du rayonnement UVX tourne de $90^{\circ}$, ce qui se traduit par une variation du signal détecté d'un facteur 2 environ, à cause de la 
différence de transmission du spectromètre. La diminution du signal à grand délai est due à la fois à la fermeture de la porte et à la diminution de l'éclairement laser pendant la porte .

Nous avons effectué des simulations basées sur un modèle simple: nous avons supposé que le signal correspondant à l'harmonique d'ordre q est donné par :

$$
S_{q}=A \int_{-\infty}^{+\infty} I_{0}(t)^{q_{-} e f f} e^{-\beta_{q} \varepsilon(t)^{2}} g(\theta) d t
$$

où $A$ est une constante de normalisation, $I_{0}(t)$ est l'intensité du laser, $q$ eff un ordre « effectif » de non-linéarité, supposé égal à 3 [13] et

$$
g(\theta)=1-\frac{1}{4}[1+\cos (2 \theta)]
$$

est une fonction qui exprime l'efficacité du spectromètre en fonction de l'orientation du plan de polarisation des harmoniques. La valeur de $\beta_{q}$ pour cette harmonique a été mesurée égale à 35 . Malgré sa simplicité, le modèle reproduit très bien les résultats expérimentaux, comme on peut le voir en Fig. 2. Pour un délai de 80 fs le modèle prévoit une diminution de la durée de l'impulsion UVX d'un facteur 3 environ, de 46,2 à 16,8 fs.

Une signature du raccourcissement de l'impulsion harmonique pourrait être l'élargissement de son spectre. Cependant celui-ci est difficile à observer du fait que les impulsions harmoniques ne sont pas limitées par la transformée de Fourier, mais présentent un chirp négatif « intrinsèque », et donc un élargissement du spectre, d'autant plus important que l'éclairement laser est élevé [14]. Or, plus la porte temporelle est étroite, moins l'éclairement pendant la génération des harmoniques est important, et donc plus l'élargissement spectral dû au chirp est faible. Ce phénomène peut effacer complètement l'effet recherché, et même amener au résultat contre intuitif d'un rétrécissement du spectre lors de l'augmentation du délai $\tau$, comme observé dans l'expérience du groupe de Lund [9]. Pour mettre en évidence le raccourcissement des impulsions harmoniques il a fallu limiter l'éclairement laser, et donc se contenter d'observer des harmoniques d'ordre relativement faible et utiliser des impulsions laser ayant un chirp positif qui compense celui des harmoniques [15]. Avec ces précautions nous avons obtenu les résultats représentés en Fig.3, qui montrent clairement un élargissement spectral des harmoniques du plateau qui ne peut être attribué qu'au confinement temporel de l'HHG.

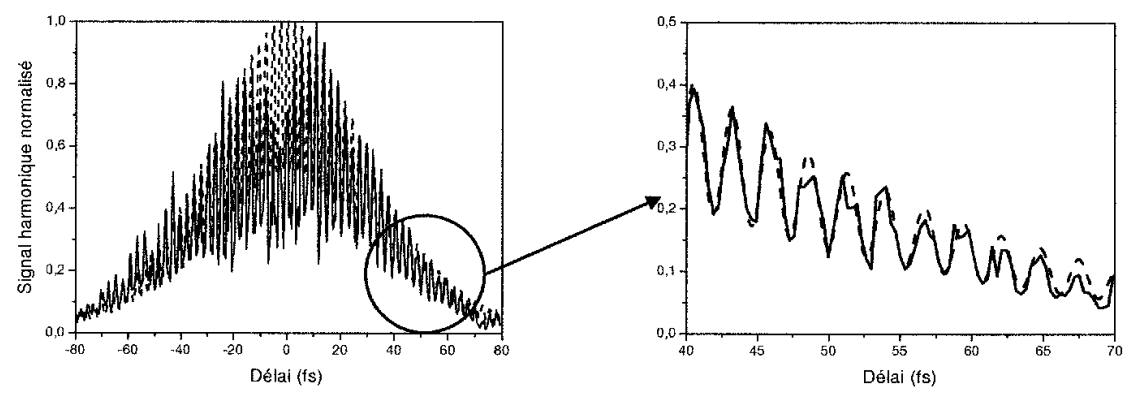

Figure 2. Variation du signal harmonique avec le délai : résultat expérimental pour la $11^{\text {ème }}$ harmonique (trait plein) et simulation (pointillés).

\section{CONCLUSIONS}

En conclusion nous avons présenté la démonstration expérimentale d'une nouvelle technique de confinement temporel de la génération d'harmoniques d'ordre élevé par manipulation de l'ellipticité du fondamental. Cette méthode peut être appliquée à la génération d'impulsions attosecondes et, 
dans un futur plus proche, à l'amélioration de la résolution temporelle d'expériences pompe-sonde dans l'UVX.

La principale limitation de cette technique réside dans le fait que, en augmentant $\tau$, d'une part l'éclairement correspondant à la porte temporelle diminue en même temps que sa durée, et d'autre part une partie croissante de l'énergie du laser ionise le gaz sans contribuer à la génération d'harmoniques. La génération d'une impulsion attoseconde isolée ne parait, par conséquent, pratiquement envisageable qu'à partir d'impulsions laser ayant une durée de 15-20 fs au plus, ce qui est néanmoins un progrès considérable par rapport aux 5-6 fs requis à présent [7].

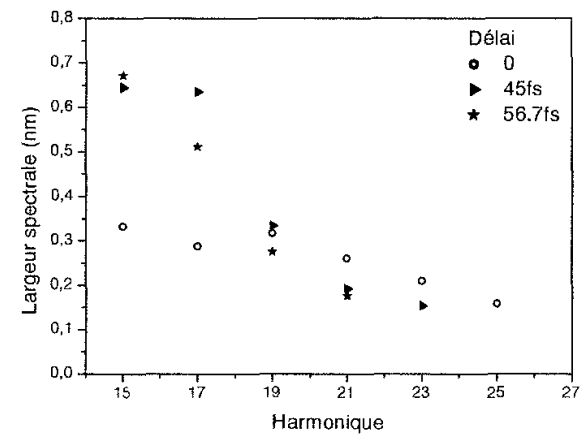

Figure 3. Largeur spectrale des harmoniques pour différentes valeurs du délai $\tau$

[1] Paul P. M., Toma E. S., Breger P., Mullot G., Augé F., Balcou Ph., Muller H. G. and Agostini P., Science 292 (2001) 1689

[2] Corkum P. B., Phys. Rev. Lett. 71 (1993) 1994

[3] Kulander C., Shafer K. J. and Krause J. L. Super Intense Laser Physics, vol. 316 NATO ASI Series B, 95 (1993)

[4] Budil K. S., Salières P., L'Huillier A., Ditmire T. and Perry M. D., Phys. Rev. A 48 (1993) R3437

[5] Antoine Ph., L'Huillier A., Lewenstein M., Salières P. and Carré B., Phys. Rev. A 53 (1996) 1725

[6] Corkum P. B., Burnett N. H. and Ivanov M. Y., Opt. Lett. 19 (1994) 1870

[7] Hentschel M., Kienberger R., Spielmann Ch., Reider G. A., Milosevic N., Brabec T., Corkum P., Heinzmann U., Drescher M. and Krausz F., Nature 414 (2001) 509

[8] Nisoli M., De Silvestri S. and Svelto O., Appl. Phys. Lett. 68 (1996) 2793

[9] Altucci C., Delfin Ch., Roos L., Gaarde M. B., L'Huillier A., Mercer I., Starczewski T. and Wahlström C. -G., Phys. Rev. A $\mathbf{5 8}$ (1998) 3934

[10] Constant E., PhD Thesis, University of Sherbrooke, Canada (1997) (non publiée)

[11] Platonenko V. T., Strelkov V. V., JOSA B 16 (1999) 435

[12] Constant E., Mevel E. and Tcherbakov O., ATTO EU workshop, Milan (2001).

[13] Wahlström C.--G., Larsson J., Persson A., Starczewski T., Svanberg S., Salières P ., Balcou Ph. and L'Huillier A., Phys. Rev. A 48 (1993) 4709

[14] Salières P., L'Huillier A. and Lewenstein M., Phys. Rev. Lett. 74 (1995) 3776

[15] Salières P., Antoine Ph., de Bohan A. and Lewenstein M. Phys. Rev. Lett. 81 (1998) 5544 